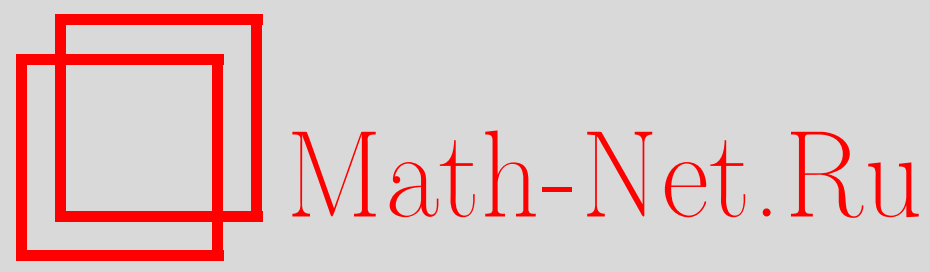

С. С. Марченков, О мощности семейства предполных классов в $P_{E}$, Матем. заметки, 2005, том 78, выпуск 6, 864-869

DOI: https://doi.org/10.4213/mzm2658

Использование Общероссийского математического портала Math-Net.Ru подразумевает, что вы прочитали и согласны с пользовательским соглашением http://www. mathnet.ru/rus/agreement

Параметры загрузки:

IP : 18.234 .197 .8

26 апреля 2023 г., 15:49:05

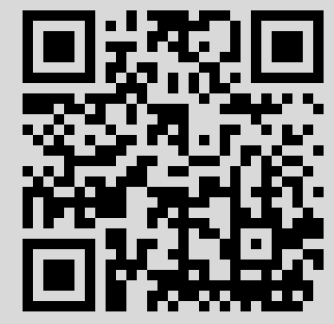




\section{О МОЩНОСТИ СЕМЕЙСТВА ПРЕДПОЛНЫХ КЛАССОВ В $P_{E}$ C. С. Марченков}

Пусть $E$ - бесконечное множество мощности $\boldsymbol{m}, P_{E}$ - множество всех функций, определенных на $E$. Доказано, что мошность семейства всех предполных в $P_{E}$ классов равна $2^{2^{m}}$. Если $C_{\mathbb{R}}$ - множество всех непрерьвных функций действительных переменных, то мощность семейства всех предполных в $C_{\mathbb{R}}$ классов равна $2^{2^{\aleph_{0}}}$.

Библиография: 13 названий.

Пусть $E$ - множество, содержащее не менее двух элементов, $P_{E}$ - множество всех функций, определенных на $E$, т.е. множество всех функций $f\left(x_{1}, \ldots, x_{n}\right)$ вида $f: E^{n} \rightarrow E$, $n=1,2, \ldots$ Множество $P_{E}$ рассматриваем как функциональную систему с операцией суперпозиции (см. [1]). Операция суперпозиции порождает на $P_{E}$ оператор замыкания $[\cdot]$; если $Q \subseteq P_{E}$, то через [Q] обозначаем множество всех функций, которые можно получить произвольными суперпозициями функций из $Q$. Множества вида $[Q]$ принято назьвать замкнутыми классами. Совокупность всех замкнутых классов из $P_{E}$ обозначим через $\mathrm{Cl}\left(P_{E}\right)$.

Множество $Q \subseteq P_{E}$ назьвается полным в $P_{E}$, если $[Q]=P_{E}$. Замкнутьй класс $Q$

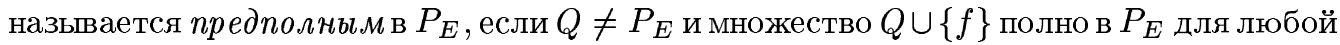
функции $f$ из $P_{E} \backslash Q$.

При изучении функциональных систем $P_{E}$ одной из основных проблем является проблема полноты: для произвольной системы функций, входящих в $P_{E}$, определить, является ли она полной в $P_{E}$. Функциональньй подход в решении проблемы полноты состоит в том, чтобы построить так называемое критериальное семейство. Совокупность $\mathscr{T}$ замкнутых классов из $\mathrm{Cl}\left(P_{E}\right)$ назьвается критериальным семейством для $P_{E}$, если произвольная система $S$ функций из $P_{E}$ полна в $P_{E}$ тогда и только тогда, когда $S$ целиком не входит ни в один из классов семейства $\mathscr{T}$.

Нетрудно видеть, что критериальньм семейством для $P_{E}$ всегда является семейство $\mathrm{Cl}\left(P_{E}\right) \backslash\left\{P_{E}\right\}$. Легко также понять, что в любое критериальное семейство обязаны входить все предполные классы. Для рассматриваемого функционального подхода оптимальньм представляется положение, когда критериальная система состоит только из предполных классов. В этом случае критерий полноты в $P_{E}$ заключается в проверке выполнения наименьшего возможного числа свойств ( свойство - это невхождение системы функций в предполньй класс). Критерии такого вида имеются для конечных множеств $E$ (см. [2]-[4]). При $|E|=2$ критериальное семейство состоит из пяти предполных

Работа выполнена при поддержке Российского фонда фундаментальных исследований, грант № 03-01-00783. 
классов [2], [3], при $|E|=3$ - из восемнадцати [5], [6]. С ростом числа $|E|$ количество предполных в $P_{E}$ классов растет примерно как двойная экспонента от $|E|[7]$.

Для бесконечных множеств $E$ исследовался лишь случай счетного $E$. Гаврилов [8] доказал, что в этом случае мошность семейства всех предполных в $P_{E}$ классов равна $2^{2^{\aleph_{0}}}$ (однако до сих пор не установлено, будет ли для счетного множества $E$ критериальное семейство состоять только из предполных классов). В работе [9] предложен некоторый метод для доказательства утверждений следующего вида: если $E$ - счетное множество, $Q$ - замкнутый класс бесконечной мощности $\boldsymbol{m}, Q \subseteq P_{E}$ и $Q$ удовлетворяет некоторым дополнительным условиям, то мощность семейства предполных в $Q$ классов равна $2^{\boldsymbol{m}}$.

В работах [10], [11] доказано, что мощность семейств предполных классов в некоторых классах функций, близких к непрерьвньм, равна $2^{2^{\aleph_{0}}}$.

В настоящей заметке установлен общий факт: для бесконечных множеств $E$ мощности $\boldsymbol{m}$ мошность семейства всех предполных в $P_{E}$ классов равна $2^{2^{m}}$. На базе тех же идей доказано, что мощность семейства всех предполных классов в классе всех непрерьвных функций действительных переменных равна $2^{2^{\aleph_{0}}}$.

Напомним, что фильтром над непустьп множеством $E$ назьвается любая непустая совокупность $F$ подмножеств множества $E$, которая удовлетворяет следующим условиЯM:

1) если $A_{1}, A_{2} \in F$, то $A_{1} \cap A_{2} \in F$;

2) если $A_{1} \in F$ и $A_{1} \subseteq A_{2} \subseteq E$, то $A_{2} \in F$;

3) $\varnothing \notin F$.

Фильтр $F$ над $E$ назьвается ультрафильтром над $E$, если $F$ не содержится ни в каком другом фильтре над $E$. Известно (см., например, [12]), что каждый фильтр над $E$ можно расширить до ультрафильтра над $E$. Кроме того, если $F$ - ультрафильтр над $E$, то для любого подмножества $A \subseteq E$ ультрафильтру $F$ принадлежит одно (и только одно) из множеств $A, E \backslash A$.

Лемма 1. Мощность семейства предполных в $P_{E}$ классов не меньше мощности семейства ультрафильтров над $E$.

ДокАЗАТЕЛЬСТВо. Для любой функции $f\left(x_{1}, \ldots, x_{n}\right)$ из $P_{E}$ положим

$$
\Delta(f)=\{x: f(x, \ldots, x)=x\} .
$$

Каждому ультрафильтру $U$ над $E$ поставим в соответствие множество функций

$$
Q_{U}=\{f: \Delta(f) \in U\} .
$$

Очевидно, что различным ультрафильтрам $U_{1}, U_{2}$ соответствуют различные множества $Q_{U_{1}}, Q_{U_{2}}$. Кроме того, поскольку ультрафильтру $U$ принадлежат не все подмножества множества $E$, имеем $Q_{U} \neq P_{E}$. Покажем, что множество $Q_{U}$ замкнуто относительно операции суперпозиции.

Пусть $f_{0}, f_{1}, \ldots, f_{m} \in Q_{U}$ и $f=f_{0}\left(f_{1}, \ldots, f_{m}\right)$ (распределение переменных в функциях $f_{1}, \ldots, f_{m}$ здесь не имеет значения). Тогда

$$
\Delta(f) \supseteq \Delta\left(f_{0}\right) \cap \Delta\left(f_{1}\right) \cap \cdots \cap \Delta\left(f_{m}\right) .
$$


Так как $\Delta\left(f_{0}\right), \Delta\left(f_{1}\right), \ldots, \Delta\left(f_{m}\right) \in U$, по свойствам 1) и 2) ультрафильтра получаем, что $\Delta(f) \in U$. Из условия $E \in U$ вытекает также, что множеству $Q_{U}$ принадлежат селекторные функции $e_{i}^{n}\left(x_{1}, \ldots, x_{i}, \ldots, x_{n}\right)=x_{i}$. Таким образом, множество $Q_{U}$ замкнуто относительно операции суперпозиции.

Докажем, что класс $Q_{U}$ предполон в $P_{E}$. Возьмем произвольную функцию $f\left(x_{1}, \ldots\right.$, $\left.x_{n}\right)$ из $P_{E}$ и произвольную функцию $g$ из $P_{E} \backslash Q_{U}$. Тогда $\Delta(g) \notin U$ и, следовательно, по свойству ультрафильтра будем иметь $(E \backslash \Delta(g)) \in U$. Пусть $h$ - какая-либо функция из $Q_{U}$ с условием $\Delta(h)=E \backslash \Delta(g)$. Положим

$$
d(x, y, z)= \begin{cases}x, & \text { если } x=y, \\ z & \text { в противном случае }\end{cases}
$$

(функция $d$ в универсальной алгебре назьвается дуальным дискриминатором [13]). Функция

$$
f_{1}\left(y, z, x_{1}, \ldots, x_{n}\right)=d\left(y, z, f\left(x_{1}, \ldots, x_{n}\right)\right)
$$

принадлежит классу $Q_{U}$. Это следует из соотношений $E \in U, \Delta(d)=E$ и $\Delta\left(f_{1}\right)=E$. Значит, функция

$$
f_{1}\left(g\left(x_{1}, \ldots, x_{1}\right), h\left(x_{1}, \ldots, x_{1}\right), x_{1}, \ldots, x_{n}\right)
$$

входит в множество $\left[Q_{U} \cup\{f\}\right]$. Нетрудно видеть, что она совпадает с функцией $f\left(x_{1}, \ldots\right.$, $\left.x_{n}\right)$, поскольку для любого $x_{1}$ из $E$

$$
g\left(x_{1}, \ldots, x_{1}\right) \neq h\left(x_{1}, \ldots, x_{1}\right)
$$

Лемма доказана.

Для любого множества $A \subseteq E$ через $\chi_{A}(x)$ обозначим характеристическую функцию множества $A$ :

$$
\chi_{A}(x)=\left\{\begin{array}{l}
1, \text { если } x \in A, \\
0, \text { если } x \notin A .
\end{array}\right.
$$

Если $A \subseteq E$, то через $\bar{A}$ обозначаем множество $E \backslash A$.

Лемма 2. Существует инбективный оператор $\Phi$ такой, что для любых различных подмно сеств $A_{1}, \ldots, A_{k}$ множсества $E$ любой непустой набор множеств из числа

$$
\Phi\left(A_{1}\right), \ldots, \Phi\left(A_{k}\right), \overline{\Phi\left(A_{1}\right)}, \ldots, \overline{\Phi\left(A_{k}\right)},
$$

не содержащий пар вида $\Phi\left(A_{i}\right), \overline{\Phi\left(A_{i}\right)}$, имеет непустое пересечение.

ДоказАтЕльство. Пусть $A \subseteq E, \chi_{A}$ - характеристическая функция множества $A$. Определим характеристическую функцию $\chi_{B}$ множества $B=\Phi(A)$.

Рассмотрим множество $E^{\prime}$, которое состоит из всевозможных элементов вида

$$
\left(\left(e_{0}, \ldots, e_{n-1}\right),\left(a_{0}, \ldots, a_{2^{n}-1}\right)\right)
$$

где $n \geqslant 1, e_{0}, \ldots, e_{n-1}$ - различные элементы из $E, a_{0}, \ldots, a_{2^{n}-1} \in\{0,1\}$, а наборы $\left(e_{0}, \ldots, e_{n-1}\right),\left(a_{0}, \ldots, a_{2^{n}-1}\right)$ предполагаются упорядоченными. Пусть $\varphi-$ взаимно однозначное отображение $E$ на $E^{\prime}$. 
Возьмем произвольньй элемент $x$ из $E$. Пусть $\varphi(x)$ имеет вид $(1)$. Положим $\chi_{B}(x)=a_{i}$, где

$$
i=\sum_{j=0}^{n-1} \chi_{A}\left(e_{j}\right) \cdot 2^{j} .
$$

Покажем, что данное определение влечет инъективность оператора $\Phi$. Пусть $A_{1}, A_{2}-$ различные подмножества множества $E$ и, например, $e_{0} \in A_{1}, e_{0} \notin A_{2}$. Возьмем такое $x$, что $\varphi(x)=\left(e_{0},(0,1)\right)$. Если $B_{1}=\Phi\left(A_{1}\right), B_{2}=\Phi\left(A_{2}\right)$, то согласно приведенному выше определению получим $\chi_{B_{1}}(x)=1$ и $\chi_{B_{2}}(x)=0$.

Если $A \subseteq E$ и $\sigma \in\{0,1\}$, то пусть

$$
\Phi^{\sigma}(A)= \begin{cases}\overline{\Phi(A)}, & \text { если } \sigma=0, \\ \Phi(A), & \text { если } \sigma=1 .\end{cases}
$$

Докажем, что для любых подмножеств $A_{1}, \ldots, A_{k}$ множества $E$ и любых значений $\sigma_{1}$, $\ldots, \sigma_{k}$ из $\{0,1\}$ множество

$$
\Phi^{\sigma_{1}}\left(A_{1}\right) \cap \cdots \cap \Phi^{\sigma_{k}}\left(A_{k}\right)
$$

непусто. С этой целью выберем в $E$ такие элементы $e_{0}, \ldots, e_{n-1}$, чтобы все множества

$$
A_{1} \cap\left\{e_{0}, \ldots, e_{n-1}\right\}, \ldots, A_{k} \cap\left\{e_{0}, \ldots, e_{n-1}\right\}
$$

оказались различными. Для любого $j, 1 \leqslant j \leqslant k$, положим

$$
i_{j}=\sum_{l=0}^{n-1} \chi_{A_{j}}\left(e_{l}\right) \cdot 2^{l} .
$$

Из несовпадения множеств (3) следует, что все числа $i_{1}, \ldots, i_{k}$ различны и, очевидно, не превосходят величины $2^{n}-1$. Пусть $\left(a_{0}, \ldots, a_{2^{n}-1}\right)$ - такой двоичньй набор, что $a_{i_{j}}=\sigma_{j}$ при $1 \leqslant j \leqslant k$ и $a_{i}=0$ при $i \notin\left\{i_{1}, \ldots, i_{k}\right\}$. Если теперь $x-$ такой элемент из $E$, что $\varphi(x)$ совпадает с (1) (для определенных вьше величин $e_{0}, \ldots, e_{n-1}$ и $a_{0}, \ldots, a_{2^{n}-1}$ ), то согласно определению оператора $\Phi$ элемент $x$ будет принадлежать множеству (2). Лемма доказана.

Теорема 1. Пусть Е - бесконечное множество мощности $\boldsymbol{m}$. Тогда мощность семейства всех предполных в $P_{E}$ классов равна $2^{2^{m}}$.

ДокАЗАтЕльство. Обозначим через $2^{E}$ булеан множества $E$. Каждому подмножеству $D \subseteq 2^{E}$ поставим в соответствие фильтр $F_{D}$ над $E$, который порождается множествами $\Phi(A)$ для $A \in D$ и $\overline{\Phi(A)}$ для $A \notin D$. Лемма 2 обеспечивает невхождение в фильтр $F_{D}$ пустого множества и, следовательно, несовпадение $F_{D}$ с булеаном $2^{E}$. Для каждого фильтра $F_{D}$ выберем ультрафильтр $U_{D}$ над $E$, которьй содержит фильтр $F_{D}$. Для различных множеств $D_{1}, D_{2}$ фильтры $F_{D_{1}}, F_{D_{2}}$ содержат взаимно дополнительные множества. Поэтому соответствующие ультрафильтры $U_{D_{1}}, U_{D_{2}}$ будут различными. Значит, мошность семейства ультрафильтров над $E$ не меньше $2^{2^{m}}$. Согласно лемме 1 получаем, что мощность семейства всех предполных в $P_{E}$ классов также не меньше $2^{2^{m}}$. С другой стороны, она, разумеется, не превосходит $2^{2^{m}}$. Теорема доказана. 
Сделаем замечание к доказанной теореме. Ее сравнительно нетрудно обобщить на другие замкнутые классы $Q \subset P_{E}$. Для этого необходимо потребовать выполнения следующих условий. Во-первых, множество

$$
\Delta(Q)=\{\Delta(f): f \in Q\}
$$

должно совпадать с булеаном $2^{E}$. Во-вторых, класс $Q$ должен выдерживать применение оператора $\Phi$. Наконец, в класс $Q$ должна входить функция $d$.

Пусть $\mathbb{R}$ - множество действительных чисел, $\mathbb{Z}$ - множество целых чисел. Через $C_{\mathbb{R}}$ обозначим множество всех непрерывных на $\mathbb{R}$ функций. Множество $C_{\mathbb{R}}$ рассматриваем как функциональную систему с операцией суперпозиции.

Лемма 3. Мощность семейства предполных в $C_{\mathbb{R}}$ классов не меньше мощности семейства ультрафильтров над $\mathbb{Z}$.

ДокАЗАТЕЛЬСТВо. Для любой функции $f\left(x_{1}, \ldots, x_{n}\right)$ из $C_{\mathbb{R}}$ положим

$$
\Delta(f)=\{x: x \in \mathbb{Z}, f(x, \ldots, x)=x\}
$$

Каждому ультрафильтру $U$ над $\mathbb{Z}$ поставим в соответствие множество функций

$$
Q_{U}=\{f: \Delta(f) \in U\}
$$

Очевидно, что различным ультрафильтрам $U_{1}, U_{2}$ над $\mathbb{Z}$ соответствуют различные множества $Q_{U_{1}}, Q_{U_{2}}$. Так же, как в лемме 1 , убеждаемся, что множества $Q_{U}$ замкнуты относительно операции суперпозиции.

Докажем, что класс $Q_{U}$ предполон в $C_{\mathbb{R}}$. Возьмем произвольную функцию $f\left(x_{1}, \ldots\right.$, $x_{n}$ ) из $C$ и произвольную функцию $g$ из $C_{\mathbb{R}} \backslash Q_{U}$ (функцию $g$ можно, очевидно, считать одноместной). Тогда $\Delta(g) \notin U$ и, следовательно, по свойству ультрафильтра будем иметь $(\mathbb{Z} \backslash \Delta(g)) \in U$. Пусть $h(x)$ - какая-либо функция из $Q_{U}$ с условием $\Delta(h)=\mathbb{Z} \backslash \Delta(g)$. Определим в классе $Q_{U}$ функцию $d_{f}\left(y_{1}, \ldots, y_{n}, z_{1}, \ldots, z_{n}, x_{1}, \ldots, x_{n}\right)$ требованиями: $\Delta\left(d_{f}\right)=\mathbb{Z}$ и

$$
d_{f}\left(g\left(x_{1}\right), \ldots, g\left(x_{n}\right), h\left(x_{1}\right), \ldots, h\left(x_{n}\right), x_{1}, \ldots, x_{n}\right)=f\left(x_{1}, \ldots, x_{n}\right) .
$$

Убедимся, что эти требования вьполнимы. Пусть $m \in \mathbb{Z}$. Если $m \notin \Delta(g)$, то пусть $\varepsilon_{m}-$ такое положительное действительное число, что

$$
|m-g(x)| \geqslant \frac{1}{2}|m-g(m)| \quad \text { при }|m-x| \leqslant \varepsilon_{m} .
$$

Аналогично, если $m \notin \Delta(h)$, то обозначим через $\varepsilon_{m}$ такое положительное действительное число, что

$$
|m-h(x)| \geqslant \frac{1}{2}|m-h(m)| \quad \text { при }|m-x| \leqslant \varepsilon_{m} .
$$

Можно считать, что для любого целого $m$ вьполняется неравенство $\varepsilon_{m}<1 / 2$.

$\Phi$ ункцию $d_{f}$ можно определить в классе $C_{\mathbb{R}}$, например, следующим образом. Пусть $m \notin \Delta(g)$. Функцию $d_{f}$ на множестве

$$
\{m\}^{n} \times \mathbb{R}^{n} \times\{m\}^{n}
$$


положим равной $m$. Далее, в точках множества

$$
\left[m-\frac{1}{2} g(m), m+\frac{1}{2} g(m)\right]^{n} \times \mathbb{R}^{n} \times\left[m-\varepsilon_{m}, m+\varepsilon_{m}\right]^{n}
$$

определим функцию $d_{f}$ так, чтобы она непрерьвно изменялась от значения $m$ в "центpe" (4) до значения $f\left(x_{1}, \ldots, x_{n}\right)$ на гранище множества (5) (к гранище множества (5) относим все те точки $\left(y_{1}, \ldots, y_{n}, z_{1}, \ldots, z_{n}, x_{1}, \ldots, x_{n}\right)$, для которых либо одно из значений $y_{1}, \ldots, y_{n}$ совпадает с $m \pm g(m) / 2$, либо одно из значений $x_{1}, \ldots, x_{n}$ совпадает с $\left.m \pm \varepsilon_{m} / 2\right)$.

Аналогично поступаем с теми значениями $m$ из $\mathbb{Z}$, для которых $m \notin \Delta(h)$. Во всех остальных случаях полагаем

$$
d_{f}\left(y_{1}, \ldots, y_{n}, z_{1}, \ldots, z_{n}, x_{1}, \ldots, x_{n}\right)=f\left(x_{1}, \ldots, x_{n}\right) .
$$

Лемма доказана.

ТЕорема 2. Мощность семейства всех предполных в $C_{\mathbb{R}}$ классов равна $2^{2^{\aleph_{0}}}$.

ДокАЗАТЕЛЬСТво почти дословно совпадает с доказательством теоремы 1. Вместо леммы 1 используем лемму 3 , а в лемме 2 в качестве множества $E$ рассматриваем счетное множество $\mathbb{Z}$. В результате получаем, что мощность семейства всех предполных в $C_{\mathbb{R}}$ классов не меньше $2^{2^{\aleph_{0}}}$. С другой стороны, мощность класса $C_{\mathbb{R}}$ равна $2^{\aleph_{0}}$. Поэтому мошность семейства всех предполньх в $C_{\mathbb{R}}$ классов не превосходит $2^{2^{\aleph_{0}}}$.

\section{СПИСОК ЦИТИРОВАННОЙ ЛИТЕРАТУРЫ}

[1] Яблонский С. В. Введение в дискретную математику. М.: Наука, 1986.

[2] Post E. L. Introduction to a general theory of elementary propositions // Amer. J. Math. 1921. V. 43. P. 163-185.

[3] Post E. L. Two-Valued Iterative Systems of Mathematical Logic. Ann. Math. Studies. V. 5. Princeton: Princeton Univ. Press, 1941.

[4] Кузнецов А. В. О проблемах тождества и функциональной полноты алгебраических систем // Труды 3-го Всесоюзного математического съезда. Т. 2. М.: Изд-во АН СССР, 1956. C. $145-146$.

[5] Яблонский С. В. О функциональной полноте в трехзначном исчислении // Докл. АН СССР. 1954. Т. 95. №6. С. 1152-1156.

[6] Яблонский С. В. Функционалные построения в $k$-значной логике // Тр. МИАН. Т. 51 , 1958. C. 5-142.

[7] Захарова Е. Ю., Кудрявцев В.Б., Яблонский С.В. О предполных классах в $k$-значных логиках // Докл. АН СССР. 1969. Т. 186. № 3. С. 509-512.

[8] Гаврилов Г. П. О функциональной полноте в счетнозначной логике // Проблемы кибернетики. Вып. 15. Наука: М., 1965. С. 5-64.

[9] Марченков С. С. О мощности множества предполных классов в некоторых классах функций счетнозначной логики // Проблемы кибернетики. Вып. 38. Наука: М., 1981. С. 109-116.

[10] Марченков С. С. О классах Слупецкого для детерминированных функций // Дискретная матем. 1998. Т. 10. № 2. С. 128-136.

[11] Марченков С. С. Функциональные аспекты проблемы полноты для некоторых классов автоматных функций // Дискретная матем. 2000. Т. 12. № 2. С. 103-117.

[12] Мальцев А.И. Алгебраические системы. М.: Наука, 1970.

[13] Fried E., Pixley A.F. The dual discriminator function in universal algebra // Acta. Sci. Math. 1979. V. 41. №1-2. P. 83-100. 